\title{
Doença venosa e sua relação com as condições de trabalho no setor de produção de refeições ${ }^{1}$
}

\author{
Venous insufficiency and its relation with \\ work conditions in the foodservice sector
}

RE S U M O

Clarissa Medeiros da Luz BERTOLDI²

Rossana Pacheco da Costa PROENÇA ${ }^{3}$

A doença venosa crônica é um problema de saúde pública importante, podendo promover inaptidão para o trabalho, tendo também uma repercussão indireta sobre a qualidade da produção e conseqüente perda de eficiência operacional. Embora ainda não haja evidência da relação direta causa-efeito de doença venosa com o trabalho, existe consenso científico de que o trabalho pode agravar o desenvolvimento da mesma. O presente estudo relata os fatores que podem influenciar o aparecimento ou agravamento de doenças venosas de membros inferiores em operadores de Unidades Produtoras de Refeições, ressaltando a importância e a possibilidade de aprofundamento do tema, o que poderia evoluir para o estabelecimento de um protocolo de prevenção e tratamento de doenças venosas em função do posto de trabalho executado. Reflete-se sobre o início de um processo de identificação deste distúrbio como uma doença de caráter ocupacional, o que contribuiria, portanto, para a reformulação conceitual dos encargos decorrentes dessa atividade profissional.

Termos de indexação: Alimentação coletiva. Condições de trabalho. Engenharia humana. Insuficiência venosa. Saúde ocupacional.

\section{A B S T R A C T}

Chronic venous disease is an important public health problem which may greatly impair the quality of one's work, generate absenteeism and hospital admittances. Although, so far, no evidence exists of the direct causeeffect relation between venous disease and work output, there is a scientific consensus that certain working conditions may increase the risk of developing the condition. The present study evaluates which factors may influence the onset or severity of lower limb venous disease in workers of a Unit of Food Service, pointing out

1 Artigo elaborado a partir da dissertação de C.M.L. BERTOLDI, intitulada "O trabalho na produção de refeições e as doenças venosas de membros inferiores". Universidade Federal de Santa Catarina; 2006.

2 Universidade Federal de Santa Catarina, Programa de Pós-Graduação em Nutrição. Campus Universitário, Trindade, 88040-900, Florianópolis, SC, Brasil. Correspondência para/Correspondence to: C.M.L. BERTOLDI. E-mail: <clarissa@intercorp.com.br>.

${ }^{3}$ Universidade Federal de Santa Catarina, Centro de Ciências da Saúde, Departamento de Nutrição. Florianópolis, SC, Brasil. 
448 | C.M.L. BERTOLDI \& R.P.C. PROENÇA

the importance and the possibility of deepening the discussions around this subject and suggest that a protocol be established to prevent and treat venous diseases stemming from working conditions. It could be the start of a process to identify this condition as an occupational disease, therefore contributing for a conceptual reformulation of the obligations stemming from this professional activity

Indexing terms: Collective feeding. Working conditions. Human Engineering. Renal Insufficiency. Occupational health.

\section{N T R O D U ÇÃ O}

O setor de produção de refeições caracteriza-se pela utilização intensiva de mãode-obra, com grande dependência do trabalho dos operadores, constituindo-se esta uma particularidade e, em algumas situações, um dos principais problemas desse segmento. Como as condições de trabalho nem sempre são favoráveis, o setor, muitas vezes, não apresenta um grande atrativo para a mão-de-obra, como observado pelos índices de rotatividade e absenteísmo que, geralmente, apresentam-se significativos ${ }^{1}$.

Embora não haja evidência da relação direta causa-efeito de doença venosa com o trabalho, existe consenso atual na opinião médica de que o trabalho pode agravar seriamente o desenvolvimento da mesma.

A doença venosa ou insuficiência venosa crônica (IVC) dos membros inferiores está entre as condições mais comuns que acometem a humanidade. Porém, o governo brasileiro passou a considerar a importância socioeconômica da doença venosa somente nos últimos anos, o que tem levado a um interesse crescente pelo conhecimento científico e clínico das questões a ela relacionadas ${ }^{2}$.

Segundo Callam³, metade da população mundial adulta apresenta sinais de doença venosa, porém menos da metade desses indivíduos apresenta varizes visíveis. No Brasil, foi encontrada a prevalência de varizes em 50,9\% das mulheres e em 37,9\% dos homens da população da cidade de Botucatu4. Ainda assim, esse é um modelo aproximado da realidade, uma vez que, na maioria dos países, os distúrbios venosos somente são registrados em caso de internação hospitalar. As varizes, por exemplo, representam apenas um aspecto da doença venosa, que se estende a graus mais avançados podendo resultar, nos casos mais graves, em úlceras de estase ${ }^{3}$.

A doença venosa é uma condição com importantes conseqüências socioeconômicas, envolvendo cuidados médicos, tanto hospitalares como domiciliares. A alta ocorrência confere-lhe o título de doença de maior incidência na população humana. É responsável por morbidade significativa, afeta a produtividade no trabalho, gerando aposentadorias por invalidez, além de restringir as atividades da vida diária e lazer ${ }^{5}$.

Apesar dos grandes avanços na área médica, como a evolução da terapêutica clínica e cirúrgica, a doença venosa é injustamente negligenciada em sua importância, seja pelo doente, seja pelos médicos não especialistas, seja pelos seguros privados médicos e até pelos médicos que trabalham em perícia na Previdência Social ${ }^{6}$. Há que considerar a existência de uma possível desvalorização/minimização da doença circulatória em função, provavelmente, de uma forte conotação estética inicial.

Estudos sugerem que uma das profissões mais afetadas pela IVC é a dos operadores de Unidades Produtoras de Refeições (UPR)7,8. Dentre os principais fatores de risco apontados, os que se relacionam com o trabalho realizado no setor de produção de refeições seriam a postura em pé, tanto em movimento estático como dinâmico, a temperatura e umidade relativas do ambiente aumentadas, o carregamento de peso inadequado, o uso de vestuário constritivo e o estresse comum no setor. O sobrepeso e obesidade parecem constituir também um fator de risco, assim como a incidência maior entre as mulheres.

As queixas dos operadores de UPRs com relação às doenças venosas geralmente são 
descritas como sensação de peso e dor, edema, câimbras noturnas e pernas cansadas. Segundo relatos, as mesmas costumam aumentar ao longo do dia, especialmente após muito tempo na posição em pé .

Frente à doença venosa, alguns aspectos relacionados às características individuais dos operadores de UPRs apresentam relevância inquestionável. Nesse sentido, encaixam-se principalmente a idade, o número de gestações e a história familiar dos indivíduos. Porém, independentemente das particularidades de cada um, quando esses operadores são submetidos às mesmas condições de trabalho que, comprovadamente, têm influência no desenvolvimento de doença venosa, quais sejam, a postura parada em pé por períodos prolongados, a temperatura e a umidade relativa do ar elevada, o carregamento de peso e a exigência de alta produtividade em condições desfavoráveis, parecem desenvolver quadros sintomáticos e clínicos positivos semeIhantes para transtornos circulatórios de membros inferiores. A partir do acompanhamento direto da jornada de trabalho, é possível identificar também outros fatores que estariam relacionados com o agravamento do quadro, como a natureza da atividade que exige um trabalho repetitivo e contínuo, constantemente sob pressão temporal, com poucas possibilidades de realizar pausas ou descansos periódicos, durante jornadas de trabalho extensas e ininterruptas ${ }^{10}$.

Além disso, condições adversas, como equipamentos danificados, utensílios difíceis de manipular, a exemplo de exaustores com funcionamento inadequado, bancadas e mesas fixas, que exigem a adoção de posturas penosas, entre outros, formam o universo de trabalho nesse setor. Diante disto, os operadores podem ser levados a buscar alternativas, por vezes, improvisadas para conseguir cumprir a sua tarefa ${ }^{10}$.

Frente ao exposto, o objetivo deste estudo é contribuir para uma caracterização mais específica dos fatores que influenciam o aparecimento ou o agravamento de doenças venosas de membros inferiores em operadores de Unidades Produtoras de Refeições.

\section{A postura em pé}

Bernardino Ramazzini, médico italiano considerado o Pai da Medicina Ocupacional, em 1700, fez a primeira referência a respeito da relação entre o trabalho na posição em pé e o desenvolvimento de varizes, na sua famosa obra De morbis artificum diatriba"1.

A postura parada em pé, comum no setor de produção de refeições, exige o trabalho estático da musculatura envolvida para manutenção dessa posição, provocando facilmente a fadiga muscular $^{12}$. Além disso, há um estrangulamento dos capilares prejudicando a circulação sangüínea e linfática. Como conseqüência, pode-se observar o aparecimento de alguns transtornos circulatórios, como varizes, varicosidades, edema e celulite.

A estase no sistema venoso é o mecanismochave da doença venosa, que também aumenta o risco de formação de coágulos e trombos. 0 mesmo mecanismo também ocorre durante o caminhar, porém em menor grau, devido à ativação da bomba da panturrilha, desde que as válvulas venosas estejam intactas. Uma vez que as mesmas estejam com defeito, caminhar acaba aumentando a pressão venosa nas extremidades inferiores por causa de uma inversão do fluxo sangüíneo. A postura em pé por períodos prolongados causa o aumento da pressão venosa, levando a um aumento da pressão de filtração capilar e, conseqüentemente, aumentando o fluxo através da membrana capilar para o espaço intersticial, provocando edema ${ }^{13}$.

Dentre os fatores determinantes das posturas adotadas no trabalho, destacam-se as exigências visuais, as exigências de precisão dos movimentos, as exigências das forças a serem exercidas, os espaços onde o operador atua e o ritmo de execução.

Estryn-Behar et al. ${ }^{14}$ usaram como marcador para classificar o trabalho em pé como penoso em função de doença venosa, o fato de permanecer trabalhando nessa posição por mais de 6 horas. 
Além das posturas parada em pé e sentada, a postura em pé em anteflexão, ou seja, inclinada para frente, também mostrou associação positiva ao desenvolvimento de insuficiência venosa ${ }^{14,15}$. Tal postura é comumente adotada na manipulação de gêneros em caixas e bacias e na higienização de utensílios em UPRs.

Com relação às posturas de trabalho, a NR $17^{(4)}$ sugere que "sempre que o trabalho puder ser executado na posição sentada, o posto de trabalho deve ser planejado ou adaptado para esta posição". Ainda, a recomendação é de que para trabalho manual sentado ou que tenha de ser feito em pé, as bancadas e mesas devem proporcionar ao trabalhador condições de boa postura, visualização e operação, com altura e características da superfície de trabalho compatíveis com o tipo de atividade, com a distância requerida dos olhos ao campo de trabalho. Além disso, devem ter características dimensionais que possibilitem o posicionamento e a movimentação adequados dos segmentos corporais ${ }^{16}$.

Além de ser comum a falta de lugares apropriados em UPRs para desenvolver atividades na postura sentada, muitas vezes observa-se uma cobrança entre os operadores de que não se deve trabalhar nessa posição em uma cozinha, pois pode significar má vontade ou preguiça. Portanto, muitos deixam de alternar suas atividades com posturas mais confortáveis por preconceito por parte dos outros operadores, evidenciando que a predominância do trabalho em pé também pode representar uma questão cultural.

\section{Temperatura e umidade relativa elevadas}

A literatura demonstra em UPRs índices de temperatura e umidade mais altos do que os recomendados para a execução do tipo de atividade envolvida9,17. Além de ser um comprometedor das condições vasculares dos operadores, é consenso, em qualquer setor produtivo, que em uma situação térmica desfavorável, com intenso calor e umidade, a produtividade diminui consideravelmente e favorece a pré-estafa e a redução da eficiência operacional.

A exposição ao calor e à umidade em UPRs é conseqüência da natureza tanto dos equipamentos utilizados quanto das atividades realizadas, e constitui componente do ambiente de trabalho que afeta direta ou indiretamente a qualidade de vida de seus operadores ${ }^{17}$. A temperatura nas áreas de cocção costuma ser alta mesmo nos meses de inverno, principalmente quando estão sendo utilizados concomitantemente o forno, os panelões a vapor e o fogão. Além disso, em muitas UPRs ocorre uma grande variação da temperatura e umidade ao longo de um dia de trabalho, em função do horário e da área da cozinha observada, principalmente dependendo dos equipamentos existentes geradores de calor e umidade.

Dentre os indivíduos contra-indicados para o trabalho em altas temperaturas, destacam-se aqueles com doenças do sistema circulatório. Mesmo que a insuficiência venosa esteja estabilizada, o trabalho em temperaturas elevadas deve ser evitado, pois o efeito do calor sobre as veias pode levar a uma vasodilatação, com agravamento do processo, e até descompensação das varizes ${ }^{18}$.

Roux et al. ${ }^{19}$, em seu estudo epidemiológico sobre doença venosa realizado com assalariados de pequenas e médias empresas de Paris (França), estabeleceram como corte para temperatura ideal entre $22^{\circ} \mathrm{C}$ e $24^{\circ} \mathrm{C}$ e para umidade relativa do ar entre $40 \%$ e $60 \%$.

O calor localizado próximo ao solo também foi considerado por alguns estudos. Pariselle et al..$^{20}$ encontraram risco relativo de 1,35 entre o trabalho sobre o chão quente e o trabalho sobre o chão frio para doença venosa, com as percentagens respectivas de sinais clínicos de 77,4\% e $57,5 \%$.

\footnotetext{
${ }^{4}$ As Normas Regulamentadoras (NR) relativas à Segurança e Medicina do Trabalho são de observância obrigatória pelas empresas privadas e públicas e pelos órgãos públicos de administração direta e indireta, bem como pelos órgãos dos poderes legislativo e judiciário, que possuam empregados regidos pela Consolidação das Leis do Trabalho (CLT).
} 
A presença significativa de vapores e gases é freqüentemente encontrada nesse setor ${ }^{21}$, pois muitas vezes os exaustores existentes, não são ligados ou não funcionam de forma adequada, prejudicando a ventilação do ambiente.

Há que considerar ainda que, além da umidade relativa do ar, outro fator agravante para IVC é a presença de umidade no piso, que, por vários momentos pode se encontrar molhado, em decorrência, entre outros, de equipamentos danificados que vazam água e de higienização das bancadas e dos fogões.

\section{Carregamento de peso}

O carregamento de peso no ambiente de trabalho, também uma constante na produção de refeições, constitui mais um fator de risco para desenvolvimento de doença venosa $a^{7,15,22}$.

As atividades desenvolvidas na produção de refeições caracterizam-se por movimentos manuais repetitivos, levantamentos de peso excessivos e permanência por períodos prolongados na postura em pé, ou mesmo em outra postura desconfortável ${ }^{9}$.

Sobaszek et al. ${ }^{15}$ e Hunzinger et al..$^{23}$ apontam como referência para carregamento de peso e risco de desenvolver doenças venosas valores iguais ou superiores a $10 \mathrm{~kg}$.

A NR 17 prevê que, com o intuito de limitar ou facilitar o transporte manual de cargas, deverão ser usados meios técnicos apropriados. Além disso, quando mulheres e trabalhadores jovens forem designados para o transporte manual de cargas, o peso máximo dessas cargas deverá ser nitidamente inferior àquele admitido para os homens, para não comprometer a sua saúde ou a sua segurança ${ }^{16}$.

Estudos demonstram que em UPR, muitas vezes, observam-se a escassez ou a inadequação de carros para transporte de cargas. Assim, pode ocorrer o transporte de caixas, latas ou panelas sem auxílio, com o agravante de que, freqüentemente, o conteúdo carregado encontra-se em alta temperatura ${ }^{24}$.

\section{Uso de vestuário constritivo}

Mekky et al. ${ }^{25}$ encontraram o uso de vestuário constritivo como uma das principais causas de varizes entre trabalhadoras inglesas da indústria têxtil.

Portanto, questões relacionadas ao vestuário dos funcionários também devem ser consideradas, pois se pode observar em UPRs o uso de calçados e vestimentas justos, o que também pode prejudicar o fluxo sangüíneo e linfático.

\section{Sexo feminino}

As deficiências circulatórias dos membros inferiores afetam mais particularmente as mulheres $4,15,20,22,23$, sendo que, em média, a cada duas mulheres, uma apresenta algum tipo de comprometimento circulatório. Este fato merece destaque, visto que a maioria dos funcionários de UPR é do sexo feminino.

A freqüência maior entre as mulheres é resultado de fatores hormonais. Porém, há que considerar que tal evidência pode ser reflexo de razões estéticas, que fazem com que as mulheres procurem tratamento três vezes mais do que os homens ${ }^{26}$.

\section{Sobrepeso e obesidade}

A literatura aponta algumas relações observadas entre sobrepeso e obesidade e a prevalência de varizes em indivíduos trabalhadores de diversos setores, evidenciando que o peso corporal constitui fator de risco para o desenvolvimento de varizes $^{3,14,15,25-27}$.

A obesidade prejudicaria as trocas do fluxo sangüíneo normal entre as veias superficiais e profundas dos membros inferiores, por causa do aumento do tecido adiposo e fibroso em volta das veias. Esse aumento prejudicaria a drenagem das veias, provocando estase e, conseqüentemente, o aparecimento de varizes ${ }^{28}$. 
Além disso, há que considerar que a influência da marcha é preponderante sobre a pressão venosa e, em indivíduos obesos, a sinergia músculo-valvular funciona de forma pouco eficiente. Primeiro, porque a marcha nesses indivíduos é menos freqüente, e segundo porque a contração muscular é menor, pois geralmente existe uma hipotonia muscular associada. O sedentarismo e a hipotonia muscular causam um déficit da circulação de retorno, gerando uma hipertensão venosa e suas conseqüências: estase e alterações capilares. Paralelamente às perturbações venosas e vênulo-capilares, localizam-se as alterações da circulação linfática ${ }^{27}$.

Matos \& Proença ${ }^{29}$ observaram que, após o início do trabalho em uma UPR, a maioria dos operadores apresentou um aumento do peso corporal. Esse aspecto é relevante, uma vez que os operadores concentravam suas refeições no local e período de trabalho. Destacam que pães, margarina, doce de fruta, biscoitos, café, leite e frutas ficam à disposição durante todo o dia na maioria das cozinhas e em um local de fácil acesso, possibilitando que os operadores comam nos intervalos das refeições.

Vários fatores agravantes de doença venosa parecem agir estatisticamente em sinergia com a obesidade. Eles não são independentes, mas ao contrário, estão ligados como conseqüência (sedentarismo, pouca atividade esportiva) ou como desencadeadores (paridade). Portanto, a obesidade aparece não como um simples fator agravante, mas em um contexto com risco de desenvolver e potencializar vários outros elementos que favorecem a doença venosa ${ }^{30}$.

\section{Condições organizacionais e cognitivas}

A organização do trabalho na produção de refeições é classicamente marcada por importante carga física e mental ${ }^{31}$, uma vez que esse setor caracteriza-se por exigir de seus funcionários alta produtividade em tempo limitado. A pressão temporal das atividades dentro de uma UPR é evidente, em função, principalmente, do fato de que as refeições devem ser consumidas no mesmo dia em que são produzidas. Essa pressão temporal é mais marcante durante os períodos que antecedem a distribuição das refeições, aos quais já foi atribuído alto índice de acidentes de trabalho. Além disso, existe uma inflexibilidade de horários, uma vez que os operadores são condicionados aos horários de distribuição das refeições, que obedecem a fatores externos à unidade'

Em relação à duração da jornada, é habitual, por exemplo, no setor de produção de refeições hospitalar, trabalhar 12 horas por 36 ou 48 horas de descanso, com número reduzido de folgas e, algumas vezes, com dois dias de trabalho seguidos, em função da substituição de colegas. Em conseqüência, observa-se um maior desgaste físico e queixas mais freqüentes relacionadas a transtornos circulatórios de membros inferiores, quais sejam dores, edema, sensação de peso, parestesia e câimbras.

Ainda, para muitos autores, a prevalência de doença venosa seja diretamente influenciada pelo número de anos passados no posto de trabalho. Pariselle et al. ${ }^{20}$ consideraram como referência para o tempo de serviço na postura em pé e sentada o período mínimo de cinco anos, e observaram a prevalência de IVC em uma razão de 1,2 .

Embora estudos demonstrem que o que norteia os operadores desse setor é a prescrição da tarefa, observa-se que os mesmos precisam assumir autonomia e responsabilidades diante de situações emergenciais, como a indisponibilidade de algum equipamento ou a falta de gêneros, sem perder de vista o objetivo final, que é a qualidade das preparações oferecidas. Lidando com essas situações emergenciais adversas dentro do ambiente de trabalho, a partir da adaptação a posturas inadequadas, por exemplo, os operadores podem ficar expostos aos fatores de risco responsáveis pelo desencadeamento ou agravamento de doenças venosas pré-existentes.

\section{CONSIDERAÇÕ ES FINAIS}

Evidencia-se que é impossível modificar alguns dos fatores de risco para a insuficiência 
venosa crônica, como idade ou história familiar, porém, outros fatores como sobrepeso e condições de trabalho podem ser alvo de ações preventivas que gerem modificações. Nesse contexto, os profissionais responsáveis pela saúde no trabalho têm um papel fundamental a desempenhar. Seria recomendável implantar medidas profiláticas, identificando, de forma sistemática, os fatores de risco e elaborando sugestões exeqüíveis para melhorar as condições de trabalho. Além do mais, é importante salientar que cabe a esses profissionais delinear estratégias preventivas e de tratamento dessa doença, ainda que a mesma não seja, até o momento, considerada de fato uma doença profissional.

A partir do quadro exposto, destaca-se a necessidade de que se desenvolvam grupos de trabalho multidisciplinares que produzam estudos para investigar a associação entre estado clínico e certos fatores de risco pessoais e ambientais para o desenvolvimento da IVC.

Assim, ressaltam-se a importância e a possibilidade da abordagem multidisciplinar ao tema, o que poderia evoluir para o estabelecimento de um protocolo de prevenção e tratamento de doenças venosas em função do posto de trabalho executado. Seria o início de um processo de identificação deste distúrbio como uma doença de caráter ocupacional, o que contribuiria, portanto, para a reformulação conceitual dos encargos decorrentes dessa atividade profissional.

\section{COLABORADORES}

C.M.L. BERTOLDI e R.P.C. PROENÇA participaram igualmente da concepção e da redação do artigo.

\section{REFERÊ N CIAS}

1. Proença RPC. Inovação tecnológica na produção de alimentação coletiva. Florianópolis: Insular; 2000.

2. Maffei FHA, Lastória S, Yoshida WB, Rollo HA. Doenças vasculares periféricas. Rio de Janeiro: Medsi; 2002.
3. Callam MJ. Epidemiology of varicose veins. $\mathrm{Br} J$ Surg. 1994; 81(2):167-73.

4. Maffei FHA, Magaldi C, Pinho SZ, Lastória S, Pinho W, Yoshida WB, et al. Varicose veins and chronic venous insufficiency in Brazil: prevalence among 1755 inhabitants of a country town. Int J Epidemiol. 1986; 15(2):210-7.

5. França LHG, Tavares V. Insuficiência venosa crônica: uma atualização. J Vasc Bras. 2003; 2(4):318-28.

6. Mello NA. Síndromes vasculares. São Paulo: Byk; 1999.

7. Tomei F, Baccolo TP, Tomao E, Palmi S, Rosati MV. Chronic venous disorders and occupation. Am J Industr Med. 1999; 36(6):653-65.

8. Banet M. Conditions de travail et maladie veineuse chez I'homme. Phlébologie. 2003; 56(2):179-82.

9. Proença R. Aspectos organizacionais e inovação tecnológica em processos de transferência de tecnologia: uma abordagem antropotecnológica no setor de alimentação coletiva. Florianópolis: Universidade Federal de Santa Catarina; 1996.

10. Bertoldi C, Proença R, Galego G, Costa S. Condiciones de trabajo en la producción de comidas como factores de riesgo para la enfermedad venosa de miembros inferiores. Medicina y Seguridad del Trabajo. 2007; 52(206): 25-32.

11. Ramazzini B. As doenças dos trabalhadores. São Paulo: Fundacentro; 2000.

12. Grandjean E. Manual de ergonomia: adaptando o trabalho ao homem. Porto Alegre: Artes Médicas; 1998.

13. Laville A. Ergonomia. São Paulo: EPU; 1977.

14. Estryn-Behar M, Berthier C, Gozlan C, Cloarec M. Apport de l'ergonomie à l'etude épidémiologique de la maladie veineus superficielle. Phlébologie. 1998; 51:15-9.

15. Sobaszek A, Dômont A, Frimat P, Dreyfus JP, Mirabaud C, Catilina P. L'insuffisance veineuse chronique des membres inférieurs en entreprise: enquête réalisée auprès de trois populations de salariés français. Arch Mal Prof. 1996; 57(3): 157-67.

16. Brasil. Ministério do Trabalho. Normas regulamentadoras do trabalho. 2005 [acesso 2005 jul 30]. Disponível em: <http://www.mtb.gov.br/legislacao/ normas_regulamentadoras/default.asp $>$.

17. Sant'Ana HMP, Azeredo RMC, Castro JR. Estudo ergonômico em serviços de alimentação. Saúde Debate. 1994; (42):45-8.

18. Mendes R. Medicina do trabalho e doenças profissionais. São Paulo: Sarvier; 1980. 
19. Roux F, Alcouffe J, Hunzinger E, Segalen $M$, Manillier P, Montéléon PY. Sensation des jambes lourdes et prévention de l'insuffisance veineuse chronique des membres inférieurs. CAMIP. 2000; 3:265-76.

20. Pariselle E, Ducord J, Cahu J, Jourdaa C, Mathieu MJ, Graille M, et al. Etude épidemiologique des troubles veineux des members inférieurs selon la posture de travail: a propos de 2.985 dossiers. Arch Mal Prof. 1992; 54:60-1.

21. Nunes BO. O sentido do trabalho para merendeiras e serventes em situação de readaptação nas escolas públicas do Rio de Janeiro. Rio de Janeiro: Fundação Oswaldo Cruz; 2000.

22. Weddell JM. Varicose veins pilot survey. $\mathrm{Br} J$ Prev Social Med. 1966; 23:179-86.

23. Hunzinger EFR, Alcouffe J, Segalen MPM, Montéléon PY. Sensation de jambes lourdes et prevention de l'insuffisance veineuse chronique des members inférieurs. Étude chez une population de salariés des petites et moyennes enterprises (PME) de l'lle-de-France. Role dês facteurs professionnels. Arch Mal Prof. 2001; 62(5): 347-406.

24. Proença R, Matos C. Condições de trabalho e saúde na produção de refeições: o estudo nas unidades de alimentação e nutrição de creches municipais de Florianópolis. Rev Ciênc Saúde. 1996; 15(1/2):73-84.
25. Mekky S, Schilling RSF, Walford J. Varicose veins in women cotton workers. An epidemiological study in England and Egypt. Br Med J. 1969; 2(5657): 591-5.

26. Krijnen RMA, Boer EM, Bruynzeel DP. Epidemiology of venous disorders in the general and occupation populations. Epidemiol Rev. 1997; 19(2):294-309.

27. Héraud G, Passas $H$. Troubles de la circulation de retour dans l'obesité. Phlébologie. 1974; 27(3): 365-74.

28. lannuzzi A, Panico S, Ciardullo AV, Bellati C, Cioffil $V$, lannuzzo $G$, et al. Varicose veins of the lower limbs and venous capacitance in postmenopausal women: relationship with obesity. J Vasc Surg. 2002; 36(5):965-8.

29. Matos CH, Proença RPC. Condições de trabalho e estado nutricional de operadores do setor de alimentação coletiva: um estudo de caso. Rev Nutr. 2003; 16(4):493-502.

30. Allaert FA, Levardon M, Vin F. Influence de l'obesité sur la maladie veineuse: étude des facteurs concomitants. Phlébologie. 1991; 44(2):271-80.

31. Guillon F, Mignee C, Chauvet JP, Renard-Marguerite $\mathrm{O}$, Proteau J. Accidents du travail chez les cuisiniers, à propos de 33 cas. Arch Maladies Professionelles. 1986; 47(3):197-8.

Recebido em: 25/6/2007

Versão final reapresentada em: 8/1/2008 Aprovado em: 23/6/2008 